SCIENTIFIC REPORT

\title{
A new donor cornea harvesting technique for posterior lamellar keratoplasty
}

\author{
O Suwan-apichon, M Rizen, J M G Reyes, S Herretes, A Behrens, W J Stark, R S Chuck
}

Br J Ophthalmol 2005;89:1100-1101. doi: 10.1136/bjo.2004.064337

\begin{abstract}
Aims: To describe a technique for posterior lamellar keratoplasty donor preparation.

Methods: In an experimental study eight human donor research corneas were mounted onto an artificial anterior chamber and deep stromal pockets dissected. Four corneas were mounted in the standard endothelial side down orientation and dissected using standard instruments (group 1). Another four corneas were mounted endothelial side up and dissected using a flat spatula (group 2). Trephined lamellar graft thickness was assessed by ultrasound pachymetry. The grafts were also analysed using vital staining of the endothelium and standard histological preparation.

Results: Achieved posterior graft thickness was 118 (SD 32) $\mu \mathrm{m}$ (group 1) and 92 (23) $\mu \mathrm{m}$ (group 2) $(\mathrm{p}=0.324)$. Percentage of devitalised endothelial cells was $0.86 \%$ (1.48\%) (group 1) and 3.9\% (2.9\%) (group 2) ( $p=0.185$ ). The dissections using both harvesting techniques remained in plane and were smooth.

Conclusions: A blunt spatula and endothelium side up orientation on an artificial anterior chamber can be used to create posterior lamellar dissections without compromising endothelial cell number or planarity when compared to standard endothelium side down harvest.
\end{abstract}

F or cases of pure endothelial damage, posterior lamellar keratoplasty (PLK) involves replacement of only the diseased endothelium along with some underlying posterior stroma, most often through a deep lamellar limbal incision, as an alternative to a full thickness penetrating graft. ${ }^{1-3}$ We describe a simplified technique, based on the approach proposed by Ignacio et $a l,{ }^{4}$ for preparing the donor cornea for PLK, and evaluate it with regard to lamellar planarity and endothelial cell loss.

\section{MATERIALS AND METHODS}

Eight corneoscleral buttons stored in Optisol GS at $4{ }^{\circ} \mathrm{C}$ and not suitable for clinical transplantation were obtained (Central Florida Lions Eye and Tissue Bank, Tampa, Florida; Donor Network of Arizona, Phoenix, AZ, USA) and divided into two groups. Ultrasonic pachymetry was used to measure the preoperative central corneal thickness after mechanical de-epithelialisation as well as after posterior lenticule removal.

Three buttons were mounted endothelial side down onto an artificial anterior chamber (Moria, Inc, France) and pressurised by air injection. Deep limbus to limbus lamellar dissections were carried out to approximately $80-90 \%$ depth using the three blade Melles lamellar keratoplasty set (Dorc, Netherlands) (group $1, \mathrm{n}=3$ ).

Three buttons were mounted endothelial side up on the same chamber, pressurised with air and dissected limbus to limbus to $10-20 \%$ depth using a $2 \mathrm{~mm}$ wide Culler iris spatula (Katena, Denville, NJ, USA) after initial entry with a $3.0 \mathrm{~mm}$ steel keratome (Sharpoint, Reading, PA, USA) (group 2, $\mathrm{n}=3$ ). The endothelium was lubricated with Optisol throughout the procedure. After removal of the corneas from the artificial anterior chamber, all six posterior
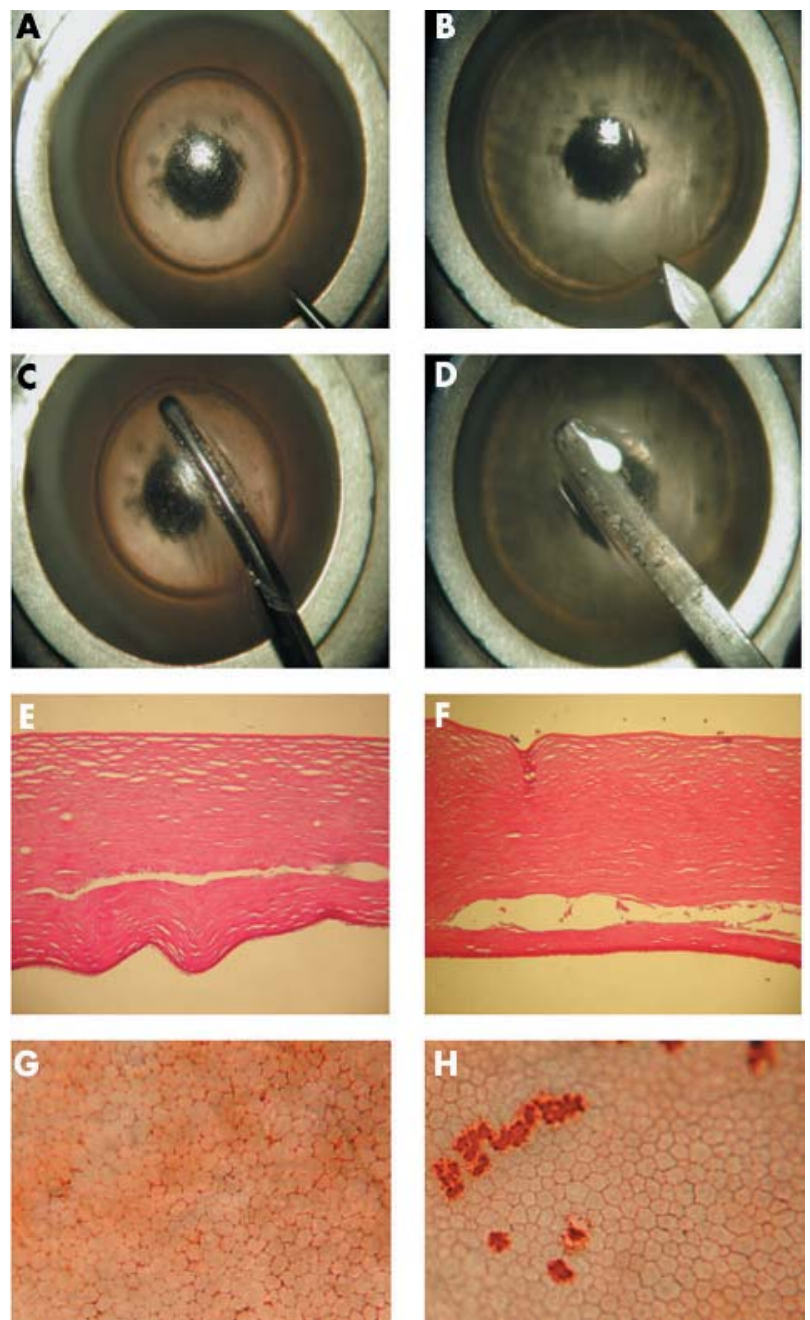

Figure 1 Comparison of two different donor cornea harvesting techniques. The left column shows a cornea mounted endothelial side down. The right column shows a cornea mounted endothelial side up. The initial entry incision is made using either the first of three Melles blades (A) or a steel keratome (B). The dissection is then performed with the second and third Melles blades (C) or a Culler iris spatula (D). Histological analysis demonstrates that the lamellar dissections are equally planar (E) and (F). Vital staining of the endothelium reveals low cell loss using both techniques $(G)$ and $(H)$. 
lenticules were then removed from the endothelial side using a $6.5 \mathrm{~mm}$ manual trephine (Surgistar, Carlsbad, CA, USA).

Endothelial cell damage after graft harvest was assessed by staining with trypan blue $0.25 \%$ (Sigma-Aldrich, Inc, St Louis, MO, USA) and alizarin red S 0.2\% (Sigma-Aldrich, Inc). ${ }^{5}$

Two corneoscleral buttons underwent stromal pocket dissection as described above but without trephination and were submitted for routine histological processing and staining with periodic acid Schiff (PAS).

\section{RESULTS}

The average harvested lenticule thickness was 118 (SD 32) $\mu \mathrm{m}$ (group 1) and 92 (23) $\mu \mathrm{m}$ (group 2) (Student's $t$ test, $\mathrm{p}=0.324)$. Percentage of devitalised endothelial cells was $0.86 \% \quad(1.48 \%) \quad$ (group 1) and $3.9 \% \quad(2.9 \%)$ (group 2) $(p=0.185)$. The dissections using each of the two harvesting techniques remained in single deep lamellar planes and were equally smooth (fig 1).

\section{DISCUSSION}

Here we describe a technique for harvesting donor tissue for posterior lamellar keratoplasty in which a corneoscleral button is mounted onto an artificial anterior chamber endothelial side up and stromal dissection is accomplished with a simple blunt spatula.

The planarity and endothelial cell loss associated with the harvest appear comparable to previously described techniques. This modified method is advantageous in that a standard re-usable blunt corneal dissector is employed without the added complexity and cost associated with sharp, disposable three knife sets. Furthermore, the orientation of the endothelium up on the anterior chamber allows greater surgeon control during the harvest.

\section{Authors' affiliations}

O Suwan-apichon, M Rizen, J M G Reyes, S Herretes, A Behrens,

W J Stark, R S Chuck, Wilmer Ophthalmological Institute, Johns Hopkins University, Baltimore, MD, USA

O Suwan-apichon, Department of Ophthalmology, Khon Kaen

University, Khon Kaen, Thailand

Support: NIH Grant EY000412-04, Stark-Mosher Center for Cataract and Corneal Diseases, Research to Prevent Blindness Inc unrestricted grant.

Correspondence to: Roy S Chuck, MD, PhD, Wilmer Eye Institute, Johns Hopkins University, 3-127 Jefferson Building, 600 North Wolfe Street, Baltimore, MD 21287-9278, USA; rchuck1@jhmi.edu

Accepted for publication 1 February 2005

\section{REFERENCES}

1 Melles GR, Eggink FA, Lander F, et al. A surgical technique for posterior lamellar keratoplasty. Cornea 1998;17:618-26.

2 Van Dooren B, Mulder PG, Nieuwendaal CP, et al. Endothelial cell density after posterior lamellar keratoplasty (Melles technique): 3 years follow-up. Am J Ophthalmol 2004;138:211-17.

3 Terry MA, Ousley PJ. Deep lamellar endothelial keratoplasty in the first United States patients: early clinical results. Cornea $2001 ; 20: 239-43$.

4 Ignacio TS, Nguyen TT, Sarayba MA, et al. A technique to harvest Descemet's membrane with viable endothelial cells for selective transplantation. Am J Ophthalmol 2005; 139:325-30.

5 Taylor MJ, Hunt CJ. Dual staining of corneal endothelium with trypan blue and alizarin red S: importance of $\mathrm{pH}$ for the dye-lake reaction. $\mathrm{Br} J$ Ophthalmol $1981 ; 65: 815-19$.

\section{Video reports}

To view the video reports in full visit our website www.bjophthalmol.com and click on the link to the video reports.

- Suture of a Subluxated Posterior Chamber Lens within the Capsular Bag. LE Fernández de Castro, KD Solomon

- Ocular Onchocerciasis: Anterior Chamber Microfilariae. WJ Flynn, HD Dillon

- Zero Phaco Microincision Cataract Surgey: The Hacc Technique. K Vaitheeswaran, $S$ Gars, R Grover, M Nadar, S Sharma

- The presenting features of multiple sclerosis. VJM Barrett, J Walker, JS Elton

- Removal of INTACS: Stepped surgical complexity demonstrated with three cases. L Ilari, J C McAlister, D S Gartry

- The Nuclear Slide: A novel approach for unleashing the potential of the hydrodissection wave. A Naseri

- Giant pleomorphic adenoma of the lacrimal gland: pre- and post-operative function. A Jain, V I Nehru, U N Saikia, C E E Reddy

- Limbal-sparing lamellar keratoplasty. S L Watson, S Rauz, J Dart 